\title{
Successful Postoperative Treatment of a Lumbosacral Ulcer in a Neonate after Myelomeningocele
}

\author{
Angéla Meszes $^{a} \quad$ Gyula Tálosi $^{b} \quad$ Krisztina Máder $^{b} \quad$ Judit Kiss $^{b}$ \\ Csilla Sánta $^{\mathrm{b}}$ Lajos Kemény $^{\mathrm{a}} \quad$ Zsanett Csoma $^{\mathrm{a}}$ \\ Departments of a Dermatology and Allergology and ${ }^{b}$ Pediatrics, University of Szeged, \\ Szeged, Hungary
}

\section{Key Words}

Myelomeningocele $\cdot$ Neonate $\cdot$ Ulcer $\cdot$ Wound management

\begin{abstract}
Wound care in neonates demands special awareness of the anatomical and physiological characteristics of their skin, and the danger of adverse mechanical and toxicological events. Here, we present the case of a full-term neonate born with myelomeningocele. Following the closing surgery on the 3rd day of postuterine life, the operated region became inflamed, the sutures opened and a necrotic discharging ulcer developed. Besides parenteral antibiotic treatment based on the microbiological findings, intelligent hydrofiber dressings were applied to the wound with regard to the special characteristics of wound care in neonates. After 72 days, the ulcer had healed with a small residual scar, and the infant is currently demonstrating normal physical and mental development.

(C) 2014 S. Karger AG, Basel
\end{abstract}

\section{Case Report}

Here, we present the case of a female neonate, born full-term (37 gestational weeks +2 days) with a normal Apgar score (9-10) and birth weight (3,250 g). After a normal vaginal delivery, a partially skin-covered fluid-filled sac $4 \mathrm{~cm}$ in diameter that protruded in the lumbosacral region was diagnosed as myelomeningocele. Physical examination revealed mild facial dysmorphia, wide nuchae and fecal incontinence, but normal lower extremity movements. The cardiological and ophthalmological examinations indicated normal functions. Neurosonography demonstrated a moderate enlargement of the temporal horn and the left lateral ventricle; abdominal sonography was negative. Due to leakage of 
Meszes et al:: Successful Postoperative Treatment of a Lumbosacral Ulcer in a Neonate after Myelomeningocele

cerebrospinal fluid from the protrusion, parenteral antibiotic treatment with cefotaxime was commenced. On the 3rd day of postuterine life, closing surgery was performed without complications. On the following day, fever, leukopenia and an elevated C-reactive protein level were observed; the antibiotic treatment was therefore supplemented with parenteral vancomycin. On the 6th day of life, the operated region became inflamed, the sutures opened and a large $(5 \times 4 \mathrm{~cm})$ necrotic ulcer with a sanguinopurulent base developed in the lumbosacral region, which spread a further 1.5-2 cm under the epidermis (fig. 1a, b). From the large amount of purulent discharge that leaked from the necrotic ulcer, ESBL-producing Klebsiella pneumoniae was cultured. Based on the microbiological findings, the antibiotic meropenem and immunoglobulin supplementation were added to the therapy. To improve the microcirculation, a parenteral infusion of pentoxifylline was administered. To counter the hypoalbuminemia and moderate peripheral edema, parenteral albumin and intravenous furosemide therapy were applied.

Wound management, performed with careful regard to the special characteristics of wound care in neonates, was started with thorough cleansing and irrigation of the wound and its surroundings with octenidine dihydrochloride-containing solution (Octenisept; $0.1 \%$ octenidine dihydrochloride $+2.0 \%$ 2-phenoxyethanol) for a few seconds followed by washing with sterile saline solution to prevent adverse toxicological events. Close attention was constantly paid to appropriate protection of the surrounding healthy skin. On the first few days, mupirocin cream was applied to the inflamed periwound, but this was later changed to a fusidic acid + hydrocortisone combination (Fucidin $\mathrm{H}^{\circledR}$ ). Because of the necrotic and infected wound base and the large amount of wound exudate, we chose an intelligent, silver-containing antiseptic hydrofiber dressing (AquaCell $\mathrm{Ag}^{\circledR}$ ) which could easily be inserted under the separated layer as well. Due to the very low silver content and low efficacy of absorption, AquaCell Ag could be used safely even in this young patient. For the second dressing, sterile gauze sheets were utilized, and the hydrofiber dressing was later combined with Versiva $\mathrm{XC}^{\circledR}$ gelling foam dressing. To fix the dressings, Mefix ${ }^{\circledR}$ self-adhesive fabric tape was used without any problems. The initial treatment included careful surgical necrectomy, and later mechanical debridement, to renew the wound base and facilitate granulation. After the wound base had become clear, we originally planned to make use of negative pressure wound therapy or split-skin graft transplantation; unfortunately, these techniques could not be used because of the extended lateral spreading of the wound. The dressings were initially changed daily because of the large amount of wound exudate. When the wound base had clarified and showed granulation from the edges, we decreased the frequency with which the dressings were changed to every 2nd or 3rd day. The dressing procedures always strictly conformed to the principles of aseptic techniques and were performed under adequate pain control. After a negative culture of the control wound exudate, the antibiotic treatment was ceased on day 21 of treatment. After 72 days of treatment, the ulcer had healed with a small, functionally nondisturbing residual scar (fig. 1d).

Due to the continuous fecal incontinence, irritative contact dermatitis with extended erosions developed in the gluteal region (fig. 2a). As the conventional zinc oxide-containing ointments (Neogranormon ${ }^{\circledR}$ and Sudocrem ${ }^{\circledR}$ ) and other hydrophobic products (white petrolatum and mineral oil) were not effective, we applied a liquid barrier film (Cavilon $3 \mathrm{M}^{\circledR}$ ) combined with Bepanthen Plus ${ }^{\circledR}$ ointment, in response to which the symptoms resolved after approximately 2 weeks (fig. $2 \mathrm{~b}$ ).

During the medical management, the cardiorespiratory and hemodynamic parameters of the neonate were always stable, and the inflammatory parameters gradually normalized in response to the systemic antibiotic treatment and local antiseptic dressings. Follow-up 
neurosonography did not show any progression in the cerebral ventricular extent. Genetic examinations revealed a normal karyotype. Enteral nutrition was commenced with good results. At 4 months of age the infant demonstrated normal physical and mental development, and her motor development is in accordance with her chronological age. The residual scar in the lumbosacral region does not cause any disturbance in physical movements. The defecation cycle has become periodic and the skin in the diaper area is normal.

\section{Discussion}

Skin care and wound management in term and preterm neonates are complicated and pose a great challenge to practitioners during neonatal care. The clinical practice of wound care in adults cannot be applied directly to these young patients in view of the anatomical and physiological differences of their skin. The immaturity of the barrier function of the epidermis results in a number of clinical complications such as an increased risk of skin traumas. Moreover, there are numerous other factors that affect wound healing such as congenital disorders, accompanying conditions and the necessary diagnostic and therapeutic procedures [1-5].

Wounds occurring in neonatal intensive care units are mostly caused by iatrogenic factors. The most common type of wounds are epidermal stripping due to adhesive dressings, extravasation injuries, surgical wounds and infections, burns due to thermal or chemical agents, excoriation in the diaper area and pressure ulcers. Additionally, wounds can develop in various congenital disorders such as aplasia cutis congenita and epidermolysis bullosa. Unfortunately, exact data are not available on the prevalence of these disorders $[1,3,6,7]$.

Evidence-based guidance for neonatal wound care practice is limited, in part due to the ethical problems of conducting clinical studies in this population. International guidelines are not available; mainly regional customs and recommendations can be found. Dressings and antiseptic agents should be administered to neonates with special awareness of the need to prevent adverse mechanical and toxicological events. The literature recommends nonadhesive and noninteractive dressings, such as hydrogels, soft silicone wound contact layers or hydrocolloids, foams, hydrofibers and semipermeable films, for routine use. Dressings applied to neonates should protect the wound and its surroundings, be easily applicable and should not need to be changed too frequently. If transparent dressings are used, the wound can readily be observed for inflammation and the quality and quantity of the wound exudates [2, 8-11].

In addition to professional local wound therapy, further essential aspects during treatment include continuous monitoring of the general status and cardiorespiratory and hemodynamic parameters, and infection control by the administration of systemic antibiotic treatment based on regular microbiological cultures.

Moreover, it should be emphasized that team work and cooperation among dermatologists, neonatologists and the nurses working in the intensive care units are crucial for the successful treatment of preterm and term neonates. 


\begin{tabular}{l|l}
\hline DOI: $10.1159 / 000360128$ & $\begin{array}{l}\text { C 2014 S. Karger AG, Basel } \\
\text { www.karger.com/cde }\end{array}$ \\
\hline
\end{tabular}

Meszes et al.: Successful Postoperative Treatment of a Lumbosacral Ulcer in a Neonate after Myelomeningocele

\section{References}

1 Baharestani MM: An overview of neonatal and pediatric wound care knowledge and considerations. Ostomy Wound Manage 2007;53:34-36, 38, 40, passim.

2 Irving V: Wound care for preterm neonates. J Neonat Nursing 2006;2:102-106.

3 Iriving V, Bethell E; on behalf of the Neonatal Advisory Group: Neonatal wound care: minimising trauma and pain. Wounds 2006;2:33-41.

4 Bree AF, Siegfried EC: Neonatal skin care and toxicology; in Eichenfield LF, Frieden IJ, Esterly NB (eds): Neonatal Dermatology. Philadelphia, Saunders, 2008, pp 59-72.

5 Gilliam AE, Williams ML: Skin of the premature infant; in Eichenfield LF, Frieden IJ, Esterly NB (eds): Neonatal Dermatology. Philadelphia, Saunders, 2008, pp 45-57.

-6 Sardesai SR, Kornacka MK, Walas W, Ramanathan R: Iatrogenic skin injury in the neonatal intensive care unit. J Matern Fetal Neonatal Med 2011;24:197-203.

7 Galbraith SS, Esterly NB: Iatrogenic and traumatic injuries; in Eichenfield LF, Frieden IJ, Esterly NB (eds): Neonatal Dermatology. Philadelphia, Saunders, 2008, pp 100-111.

8 Neonatal Intensive Care Unit: Skincare Guidelines for Babies in NICU. Guidelines of John Hunter Children's Hospital, document No. JHCH_NICU_3.05., 2011. www.kaleidoscope.org.au/site/content.cfm?page.

9 National Guideline Clearinghouse (NGC): Neonatal Skin Care, ed 2. Evidence-based clinical practice guideline. Rockville, Association of Women's Health, Obstetric and Neonatal Nurses AWHONN, Agency for Healthcare Research and Quality (AHRQ), 2007. www.kaleidoscope.org.au/docs/gl/skincare_nicu.pdf.

10 Liverpool Women's NHS Foundation Trust: Skin/wound care guidelines (version 7-NICU83). 2011. http://www.liverpoolwomens.nhs.uk/Library/health_professionals/Neonatal_policy_library/Skin\%20and $\% 20$ wound\%20care.pdf.

11 Issues in Neonatal Wound Care: Minimising Trauma and Pain. Mölnlycke Health Care. 2005. http://www.liverpoolwomens.nhs.uk/Library/health_professionals/Neonatal_policy_library/Skin\%20and $\% 20$ wound\%20care.pdf. 


\section{Case Reports in Dermatology}

\begin{tabular}{l|l}
\hline DOI: $10.1159 / 000360128$ & $\begin{array}{l}\text { C 2014 S. Karger AG, Basel } \\
\text { www.karger.com/cde }\end{array}$ \\
\hline
\end{tabular}

Meszes et al:: Successful Postoperative Treatment of a Lumbosacral Ulcer in a Neonate after Myelomeningocele
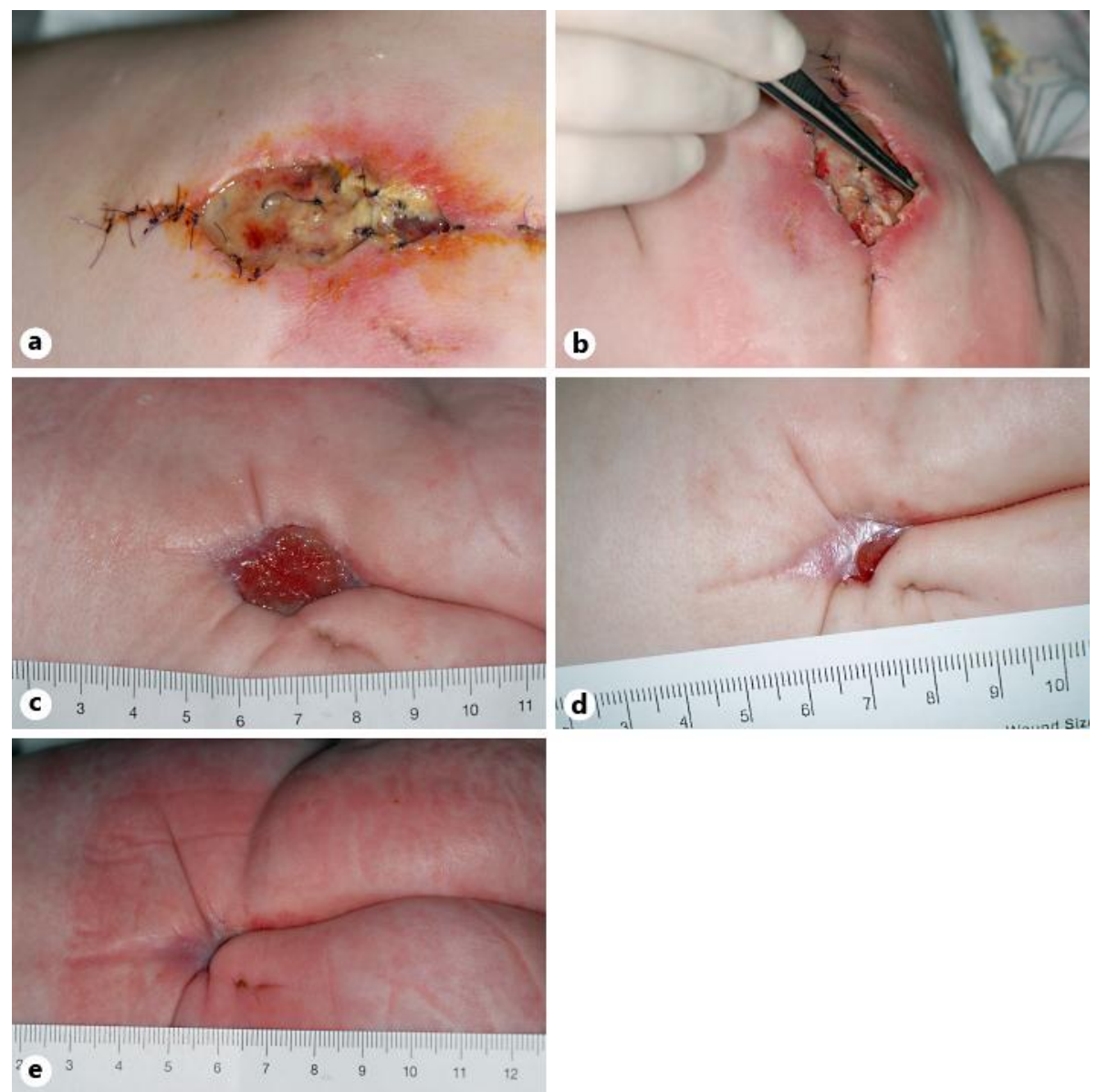

Fig. 1. a, b Necrotic, superinfected, exudating ulcer with inflamed surroundings, spreading $1.5-2 \mathrm{~cm}$ under the epidermis after the opening of the sutures. c After 1 month: clear, granulating tissue on the wound base, with a normal periwound. d After 2 months: epithelization from the fixed edges of the wound. e After 72 days: an epithelized wound with a residual scar $1 \mathrm{~cm}$ in diameter.
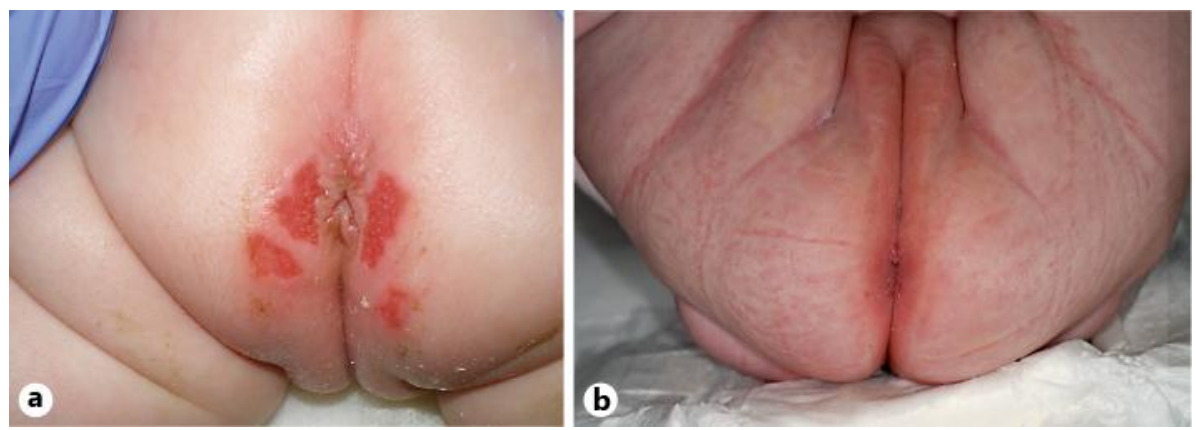

Fig. 2. a Irritative contact dermatitis with erosions due to fecal incontinence in the diaper area. b After treatment, normal skin can be seen in the diaper area. 